\section{Gutes und böses Wachstum}

\author{
In der Debatte um das Wachstum sind die Fronten verhärtet. \\ Endlicher Planet gegen Kapitalismus. Es ist Zeit für einen \\ Kompromiss. Umsatzwachstum lässt sich in Preis- und Mengen- \\ wachstum trennen. Nur Letzteres hat aber verheerende Aus- \\ wirkungen auf den Ressourcenverbrauch. Warum aber sollten \\ die Unternehmen nicht nach Preiswachstum streben? \\ Von Robert Mende-Kremnitzer
}

A uch die Natur hat es mit Mengenwachstum versucht. Aus dem Einzeller haben sich in der Urzeit riesige Pflanzen und Tiere entwickelt. Doch im Wettbewerb um die Ressourcen haben sich schließlich die schlauen und schnellen Spezies durchgesetzt, nicht die großen.

Wachstum ist nicht gleich Wachstum. Auch nicht in der Wirtschaft. Im Kapitalismus zählt das Umsatzwachstum. Umsätze können aber auf zwei Arten wachsen - über die verkaufte Menge oder über den verrechneten Preis.

\section{Wachstum ist nicht gleich Wachstum}

Mengenwachstum ist immer auch ein Mehr an Ressourcenverbrauch. Auch wenn der Effizienzgrad mit der Produktionsmenge in der Regel zunimmt, so steigen bei den meisten Produkten die direkten Material- und Ressourcenverbräuche proportional zur Produktionsmenge. Preiswachstum andererseits ist zunächst einmal Qualitätswachstum, vorausgesetzt wir unterstellen halbwegs vollkommene Märkte, auf denen der Wert eines Angebots für den Kunden über den dafür bezahlten Preis definiert wird.

Wird Umsatzwachstum durch stetig steigende Produktqualitäten bei gleichen Absatzmengen erzielt, dann führt das per se nicht zu einem höheren Verbrauch an Ressourcen. Es gibt also aus ökologischer Perspektive ein „gutes“ Preiswachstum und ein „schlechtes“ Mengenwachstum.

Eine kleine Stichprobe von sieben Geschäftsberichten von großen deutschen und österreichischen Unternehmen aus den sechs Jahren nach der Finanzkrise 2008 zeigt, dass das aktuelle Wirtschaftswachstum zum größten Teil auf Mengenwachstum basiert und nur zu einem kleinen Teil auf nominalem Preiswachstum. Wird das nominale Preiswachstum noch dazu um die Inflationsrate bereinigt, dann zeigt sich, dass der reale Wert der Produkte in den letzten sechs Jahren bei fast allen untersuchten Unter- nehmen abnahm. Ob das die richtigen Strategien für das 21. Jahrhundert sind?

Der Index des Preiswachstumsanteils zeigt, wie stark das Unternehmen durch eine Steigerung von Mengen oder Qualität wächst. Dieser Anteil ist das „gute“ Wachstum und stellt die ökologische Nachhaltigkeit des Wachstums dar. Markt- und Mengenerweiterung ist oft einfacher und schneller umsetzbar als Leistungserweiterung. Noch dazu steigt das Risiko der Produktentwicklung mit dem Neuigkeitsgrad und der Dauer der Entwicklung (Engeln 2006).

Darüber hinaus treibt auch die Suche nach Kosteneffizienz die Unternehmen zu Mengenwachstum. Über die Skalierung von Bezugs- oder Herstellkosten erarbeitet sich das Management Spielraum für die Preisgestaltung.

\section{Neue Ziele für Unternehmen}

Der Ausstieg aus der Spirale von Mengen, Kosten und Umsatz wird einen völlig neuen Zugang zu unternehmerischen Zielsetzungen erfordern. Qualitätswettbewerb muss über Mengenwettbewerb gestellt werden. Der Preiswettbewerb wird nach oben geführt werden. Wer das im Sinne der Kunden bessere, also teurere Produkt hat, gewinnt den Kunden. Absatzmenge muss dagegen als Unternehmensziel in den Hintergrund treten. Derzeit arbeiten wachstumskritische Unternehmer bereits in dieser Richtung. Die knappe Ressource Zeit wird dabei gerne in die Weiterentwicklung der Produkte, statt in die mengenorientierte Markterweiterung investiert (Liesen et al. 2013).

\begin{tabular}{|c|c|c|c|c|c|}
\hline Periode 2008-2013 & $\begin{array}{l}\text { Umsatz- } \\
\text { wachstum }\end{array}$ & $\begin{array}{l}\text { Mengen- } \\
\text { wachstum }\end{array}$ & $\begin{array}{l}\text { Preis- } \\
\text { wachstum } \\
\text { nominal }\end{array}$ & $\begin{array}{l}\text { Preis- } \\
\text { wachstum } \\
\text { real }\end{array}$ & PWA* \\
\hline Lenzing AG Div. Faser & $58,3 \%$ & $64,8 \%$ & $-4,0 \%$ & $-11,0 \%$ & $-6,8 \%$ \\
\hline BMW AG & $44,8 \%$ & $37,7 \%$ & $5,2 \%$ & $-10,1 \%$ & $11,5 \%$ \\
\hline Flughafen Wien AG & $13,5 \%$ & $11,7 \%$ & $1,6 \%$ & $-9,2 \%$ & $12,1 \%$ \\
\hline Rosenbauer AG & $46,9 \%$ & $33,6 \%$ & $9,9 \%$ & $-9,2 \%$ & $21,1 \%$ \\
\hline Volkswagen AG & $55,3 \%$ & $34,5 \%$ & $15,5 \%$ & $4,7 \%$ & $28,0 \%$ \\
\hline AMAG AG & $52,0 \%$ & $16,3 \%$ & $30,6 \%$ & $21,0 \%$ & $58,9 \%$ \\
\hline Mayr Melnhof AG Div. Karton & $10,0 \%$ & $3,4 \%$ & $6,4 \%$ & $-4,3 \%$ & $64,3 \%$ \\
\hline
\end{tabular}

Tabelle 1: Wachstumszahlen ausgewählter Unternehmen von 2008 bis 2013.

Quelle: Geschäftsberichte der Unternehmen

\section{Literatur \\ Engeln, W. (2006): Methoden der Produktentwick- lung. Oldenbourg Industrieverlag. \\ Liesen, A., et al. (2013): Wachstumsneutrale Unter- nehmen. In: Schriftenreihe des IÖW 205/13.}

\section{AUTOR + KONTAKT}

Robert Mende-Kremnitzer lehrt seit 14 Jahren Controlling und Nachhaltigkeit an der Fachhochschule Joanneum in Graz, Österreich.

Robert Mende-Kremnitzer, Tel.: +43 664 1843520, E-Mail: robert.kremnitzer@fh-joanneum.at
No Derivates License (http://creativecommons.org/licenses/by-nc-nd/4.o/ deed.de), which permits copying and redistributing the material in any medium or format, provided the original work is properly cited, it is not used for commercial purposes and it is not remixed, transformed or built upon. 